\title{
ВЫСОКОСКОРОСТНОЙ КОММУНИКАЦИОННЫЙ ИНТЕРФЕЙС МОЗГ-КОМПЬЮТЕР НА ОСНОВЕ КОДИРОВАННЫХ ЗРИТЕЛЬНЫХ ВЫЗВАННЫХ ПОТЕНЦИАЛОВ
}

\author{
Р. К. Григорян ${ }^{1 凶}$, Д. Б. Филатов ${ }^{1,2}$, А. Я. Каплан
}

1 Биологический факультет, Московский государственный университет имени М.В.Ломоносова, Москва, Россия

Механико-математический факультет, Московский государственный университет имени М. В. Ломоносова, Москва, Россия

Технологии интерфейсов мозг-компьютер (ИМК) активно используют в клинической практике для обеспечения коммуникации с пациентами, не способными к речи и движениям. Ввод команд в компьютер посредством таких интерфейсов осуществляют на основе выделения в ЭЭГ вызванных потенциалов в ответ на значимые для пользователя стимулы, например подсвеченные на экране буквы. Целью работы было исследовать показатели ЭЭГ и эффективность ввода команд в перспективном типе ИМК на основе так называемых кодированных вызванных потенциалов, возникающих в ответ на определенную последовательность подсветок нужной буквы. На 15 здоровых добровольцах изучали работу такого интерфейса на разных скоростях подачи стимульных последовательностей при их инвертировании, когда подсветку и ее отсутствие меняли местами. Показано, что оптимальное значение скорости стимуляции имеет значительную индивидуальную вариабельность, а инверсия стимульной последовательности не оказывает влияния на работу интерфейса. Медианная точность выбора команд составила: в режимах с циклом стимуляции 1 с - 0,96 и 0,95 (скорость передачи информации 142 и 141 бит/мин); в режиме со стимульным циклом 2 с - 1; с циклом 0,5 с - 0,33. Максимальную выраженность вызванных потенциалов наблюдали в отведении Oz. Сделано предположение о том, что оптимизация нейроинтерфейсов на основе кодированных вызванных потенциалов возможна на основе индивидуального подбора параметров стимуляции

Ключевые слова: нейроинтерфейсы, вызванные потенциалы, ЭЭГ, электроэнцефалограмма, ИМК, интерфейс мозг-компьютер, нарушения речи, КЗВП, кодированные зрительные вызванные потенциалы

Информация о вкладе авторов: Р. К. Григорян - планирование и проведение эксперимента, обработка данных, подготовка статьи; Д. Б. Филатов планирование эксперимента, разработка программного обеспечения (ПО), подготовка статьи; А. Я. Каплан - постановка задачи, планирование эксперимента, руководство проведением исследования, подготовка статьи.

Соблюдение этических стандартов: исследование одобрено этической комиссией Биологического факультета МГУ имени М. В. Ломоносова (протокол № 29-ч от 11 декабря 2015 г.); испытуемые подписали добровольное информированное согласие на участие в исследовании.

Для корреспонденции: Рафаэль Каренович Григорян

ул. Ленинские горы, д. 1, стр. 12, г. Москва, 119234; grraph.bio@gmail.com

Статья получена: 29.07.2018 Статья принята к печати: 24.03.2019 Опубликована онлайн: 04.04.2019

DOI: $10.24075 /$ vrgmu.2019.019

\section{HIGH-SPEED BRAIN-COMPUTER COMMUNICATION INTERFACE BASED ON CODE-MODULATED VISUAL EVOKED POTENTIALS}

Grigoryan $\mathrm{RK}^{1} \otimes$, Filatov $\mathrm{DB}^{1,2}$, Kaplan $\mathrm{AY}^{1}$

${ }^{1}$ Faculty of Biology, Lomonosov Moscow State University, Moscow, Russia

2 Department of Mechanics and Mathematics, Lomonosov Moscow State University, Moscow, Russia

Brain-computer interface $(\mathrm{BCl})$ technologies are actively used in clinical practice to communicate with patients unable to speak and move. Such interfaces imply identifying potentials evoked by stimuli meaningful to the patient in his/her EEG and interpreting these potentials into inputs for the communication software. The stimuli can take form of highlighted letters on a screen, etc. This study aimed to investigate EEG indicators and assess the command input performance of a promising type of $\mathrm{BCl}$ utilizing the so-called code-modulated visual evoked potentials (CVEP) appearing in response to a certain sequence of highlights of the desired letter. The operation of the interface was studied on 15 healthy volunteers. During the experiments, we changed the speed of stimuli demonstration and inverted the order of flashing. It was established that the optimal speed of stimulation significantly depends on individual traits of the person receiving the stimuli, and inversion of their sequence does not affect operation of the interface. The median accuracy of selection of commands was as follows: $1 \mathrm{~s}$ stimulation cycle mode -0.96 and 0.95 (information transfer rate 142 and 141 bit per minute); $2 \mathrm{~s}$ stimulation cycle mode $-1 ; 0.5 \mathrm{~s}$ cycle -0.33 . The evoked potentials were most expressed at the $\mathrm{Oz}$ site. It was assumed that CVEP-based brain-computer interfaces can be optimized through individualization of the set of stimulation parameters.

Keywords: neurocomputer interfaces, evoked potentials, EEG, Electroencephalogram, BCl, brain-computer interfaces, speech disorders, CVEP, code-modulated visual evoked potentials

Author contribution: Grigoryan RK - experiment planning and conducting, data processing, article authoring; Filatov DB — experiment planning, software development, article authoring; Kaplan AY — task setting, experiment planning, general research effort management, article authoring.

Compliance with ethical standards: the study was approved by the ethical commission of the Lomonosov Moscow State University's Faculty of Biology (Protocol № 29-ch of December 11 2015); the participants signed a voluntary informed consent.

Correspondence should be addressed: Rafael K. Grigoryan Leninskie Gory 1, bld. 12, Moscow, 119234; grraph.bio@gmail.com

Received: 29.07.2018 Accepted: 24.03.2019 Published online: 04.04.2019

DOI: $10.24075 /$ brsmu.2019.019

Интерфейс мозг-компьютер (ИМК) - это технология, позволяющая пациентам с нарушениями речи и движений управлять компьютером посредством анализа коррелятов нервной деятельности. Использование ИМК требует от пользователя фокусирование внимания либо на внутренних образах, например, движениях собственной конечности, либо на экранных объектах, в частности, нужных в данный момент буквах, и выделения специфических ЭЭГ-маркеров этих ментальных действий [1-3]. Интерфейсы на основе зрительных потенциалов, вызываемых, к примеру, подсветками экранных объектов, позволяют детектировать по ЭЭГ большое число команд, соответствующих числу стимульных экранных 
объектов. В традиционном исполнении этой технологии в качестве ЭЭГ-маркера внимания пользователя к нужному ему объекту используют компонент ПЗО0, возникающий на подсветку именно этого объекта, например нужной буквы $[4,5]$. В последнее время подобные нейроинтерфейсы входят в практику медицинской реабилитации в качестве устройств для замещения коммуникации у пациентов с тяжелыми нарушениями речи и движений [6]. Эти системы обеспечивают достаточно высокую надежность выбора команд когнитивно сохранными пациентами, однако имеют недостаток - сравнительно низкую скорость работы. Так, в сравнительном исследовании работы ИМК у пациентов с боковым амиотрофическим склерозом и у здоровых испытуемых было показано, что скорость печати текста не поднималась выше 2-3 букв в минуту при 14-кратном повторе каждого целевого объекта. При достигнутой обеими группами точности больше 95\% это соответствует скорости передачи инсоормации 11-14 бит/мин [7]. Такая низкая скорость передачи информации посредством ИМК не может обеспечить комфортное использование нейроинтерфейсов на основе компонента Пзо0 даже здоровыми людьми. Новые перспективы в повышении скорости нейроинтерфейсов открывает использование в ИМК кодированных зрительных потенциалов (КЗВП) - совокупной реакции ЭЭГ на специальные последовательности подсветок нужного экранного объекта с неравными интервалами. В результате стимуляции такой последовательнстью в ЭЭГ регистрируется зрительный вызванный потеницал стабильного состояния. Этот вызванный потенциал синхронизирован по фразе со стимуляцией, так же как и при стимуляции с постоянной частотой, которая лежит в основе ИМК на основе SSVEP (steady-state visual evoked potentials). За счет синхронизации по фазе вызванный потенциал до некоторой степени сохраняет свойства стимульной последовательности, в частности цикличность, автокорреляционные и спектральные характеристики. Если использовать для презентации нескольких зрительных стимулов несколько разных последовательностей, которые будут минимально коррелировать между собой, то можно различать вызванные потенциалы, соответствующие разным стимулам при помощи корреляционного анализа. Существуют различные наборы бинарных последовательностей с подходящими кросскорреляционными свойствами, такие как коды Голда, коды Баркера и m-последовательности. Всех их применяют для разделения сигналов на одной несущей частоте в различных приложениях, в частности в мобильной связи и спутниковой навигации.

М-последовательность - это псевдослучайная двоичная последовательность, одной из особенностей которой является наличие единственного пика автокорреляционной функции при нулевом сдвиге. Одна $m$-последовательность способна при циклическом сдвиге породить несколько последовательностей, не скореллированных между собой. Эта особенность облегчает ее использование в ИМК при большом количестве стимулов, так как позволяет сократить длительность обучения классификатора. Для различения вызванных потенциалов, соответствующих разным стимулам, необходимо собрать обучающую выборку с потенциалами, возникающими в ответ на каждый стимул. При использовании для каждого стимула своей бинарной последовательности, порожденной одной $m$-последовательностью, достаточно получить эталонный вызванный потенциал только на нее, после чего обнаруживать целевой стимул по сдвигу пика корреляционной функции между пространственно отфильтрованными участками записываемой ЭЭГ и эталонным вызванным потенциалом. В результате продолжительность обучения не зависит от количества разных стимулов, что позволяет использовать достаточное для печати текста количество стимулов.

С помощью канонического корреляционного анализа в ЭЭГ можно при небольшом числе повторов циклов активации стимулов (не более 2-3) с высокой надежностью обнаружить короткие эпизоды кодированных вызванных потенциалов, синхронных с подсветками нужного на данный момент экранного объекта. Такие ИМК могут обеспечивать скорость передачи информации более 100 бит/мин [8]. Кодированные вызванные потенциалы могут быть получены как на ЭЭГ, так и на электрокортикограмме [9] и использоваться для оптимизации работы ИМК с учетом потенциалов, связанных с ошибками [10], а также с использованием вариаций цветовой гаммы в стимульной среде [11].

С целью поиска оптимальных режимов кодированных подсветок экранных объектов в ИМК в настоящей работе тестировали работу оператора ИМК при различных последовательностях подсветок стимулов, а также при разной скорости их подачи.

\section{МАТЕРИАЛЫ И МЕТОДЫ}

\section{Испытуемые}

В исследовании приняли участие 15 здоровых добровольцев (7 женщин и 8 мужчин) в возрасте 18-30 лет. Критерии Включения пациентов в исследование: отсутствие истории неврологических заболеваний, в том числе эпилепсии; нормальное или скорректированное зрение. Критерии исключения: иной возраст; неврологические заболевания в анамнезе; проблемы со зрением.

\section{Регистрация ЭЭГ}

Запись биопотенциалов производили с помощью усилителя ЭЭГ «Нейровизор БММ» (Медицинские Компьютерные Системы; Россия). Использовали 22 отведения (FCz, C3, C1, Cz, C2, C4, CP3, CP1, CPz, CP2, CP4, P5, P3, Pz, P4, P6, PO3, POz, PO4, O1, Oz, O2) с AFz в качестве заземляющего электрода и двух усредненных ушных электродов в качестве ресерента. Перед записью производили проверку межэлектродного сопротивления, запись начинали после того, как сопротивление было доведено до значений не более 10 КОм. Частота дискретизации составляла 500 Гц.

\section{Экспериментальная установка}

Управление экспериментом производили с компьютера с помощью специально разработанного авторами программного обеспечения (ПО) на языке $\mathrm{C}++$. Стимулы демонстрировали испытуемому на 24-дюймовом мониторе с частотой обновления 120 Гц. Испытуемый располагался в кресле на расстоянии примерно 60 см от монитора. Для обеспечения синхронности записи ЭЭГ и представления стимулов был использован фотодатчик.

\section{Визуальная среда}

Испытуемому предъявляли визуальную среду, состоявшую из 32 квадратных ячеек с буквами на черном фоне, 
организованными в виде таблицы 4 × 8. В качестве стимуляции использовали смену цвета стимула ячейки с черного на белый.

Алгоритм смены цвета определяли $m$-последовательностью длиной 63 бита. Каждая ячейка с буквой меняла цвет в соответствии со своей $m$-последовательностью, полученной из базовой путем последовательного циклического сдвига на 2 бита. Таким образом, последовательность активаций первой ячейки была базовой, вторая была сдвинута на 2 бита, десятая на 18 и так далее. Всего были использованы две базовые $m$-последовательности: базовая - [0, 0, 0, 0, 0, $1,1,1,1,1,0,1,1,1,1,0,0,1,1,1,0,1,0,1,1,0,0,0,0,1,0,1,1$, $1,0,0,0,1,1,0,1,1,0,1,0,0,1,0,0,0,1,0,0,1,1,0,0,1,0,1,0$, 1, 0] и инвертированная - $[1,1,1,1,1,0,0,0,0,0,1,0,0,0,0$, $1,1,0,0,0,1,0,1,0,0,1,1,1,1,0,1,0,0,0,1,1,1,0,0,1,0,0,1$, $0,1,1,0,1,1,1,0,1,1,0,0,1,1,0,1,0,1,0,1]$.

Других m-последовательностей длиной 63 бита, не являющихся циклическими сдвигами этих, нет. Инвертированная последовательность аналогична базовой с точки зрения автокорреляционных свойств, но порождает существенно отличающуюся зрительную стимуляцию.

В ходе эксперимента испытывали четыре режима работы ИМК, различавшихся самими $m$-последовательностями и скоростью стимуляции. В первых двух режимах стимуляции использовали, соответственно, базовую и инвертированную последовательность. Период составлял 1 с. В третьем режиме («медленном») использовали базовую последовательность с периодом в 2 с, а в четвертом («быстром») - с периодом в 500 мс.

Таким образом, длительность реализации одного бита в белом и черном цветах для стандартной, быстрой и медленной скоростей стимуляции составляла примерно 16, 8 и 32 мс соответственно.

\section{Структура исследования}

Каждый испытуемый принял участие в четырех экспериментальных сессиях. После инструктажа и установки электродов случайным образом определяли порядок режимов в исследовании. Каждый режим начинался с этапа обучения классификатора, для чего испытуемому предлагали смотреть на один из стимулов в течение 40 полных периодов предъявления последовательности. После этого испытуемый должен был, в заранее определенном порядке концентрируясь на стимулах, ввести 32 команды. Для ввода команды нужно внимательно смотреть на определенную букву и концентрироваться на ее подсветках. Через несколько секунд система дает ответ, который может быть как правильным, так и неправильным, после чего стимуляцию останавливают. После перерыва в несколько секунд она начинается снова, при этом испытуемый пытается ввести другую команду.

Решение о вводе команды принималось при достижении классификатором определенного порога. Точность выбора команд определяли как соотношение правильно введенных команд к общему количеству попыток ввода.

\section{Классификация паттернов}

Канонический корреляционный анализ позволяет получить веса каналов для пространственной фильтрации ЭЭГ и выделения существенного отклика на стимульную последовательность. Веса, полученные в результате анализа ЭЭГ, записанные в режиме обучения, используют для уменьшения размерности сигнала. Результатом этапа обучения является одноканальный усредненный по 40 полным периодам сигнал отклика на $m$-последовательность. При работе онлайн после поступления одномерного сигнала, соответствующего предъявлению полной $m$-последовательности, строится его корреляционная функция с сигналом, полученным при обучении. Выбранная пользователем команда определяется по смещению пика этой корреляционной функции. Для определения номера целевого стимула достаточно разделить временной сдвиг максимума корреляционной функции на время реализации одного бита и сдвиг между последовательными стимулами.
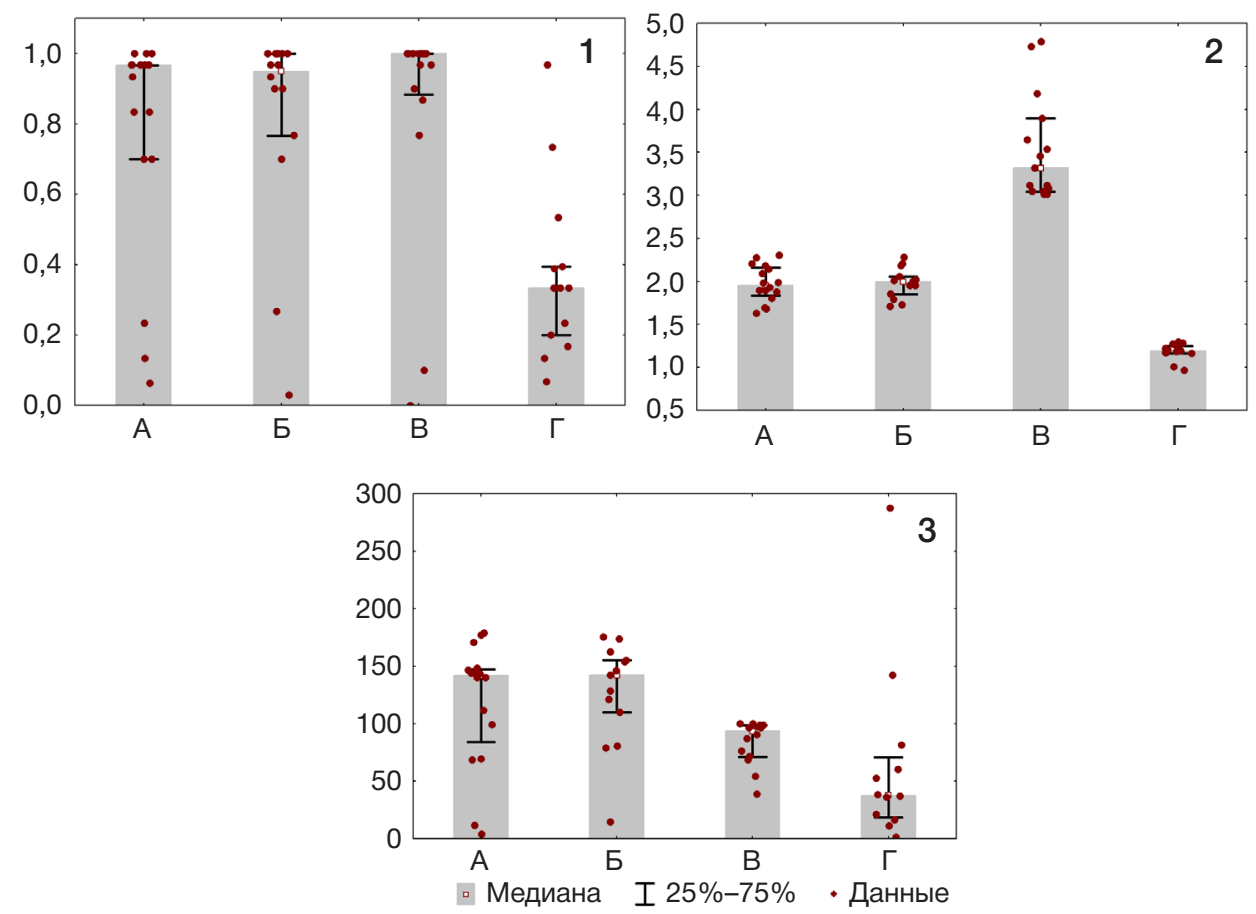

Рис. 1. Показатели работы пользователей в нейроинтерфейсе. 1 - точность ввода команд; 2 - среднее время ввода команд (секунды); 3 - скорость передачи информации, бит/мин; А — базовая стимульная последовательность; Б — инвертированная; В — медленный режим; Г — быстрый режим 


\section{Обработка результатов}

Обработку результатов производили с помощью пакета scipy 1.1.0 [12]. Для построения корреляционных карт применяли нормализованную кросс-корреляцию. Для попарных сравнений применяли критерий Уилкоксона с поправкой Хольма-Шидака на множественные сравнения.

\section{РЕЗУЛЬТАТЫ ИССЛЕДОВАНИЯ}

\section{Оценка точности классификации и скорости передачи информации}

Медианная точность выбора команд в ходе эксперимента достигала 1 в медленном режиме с периодом m-последовательности в 2 с (рис. 1). В режимах с базовой и инвертированной $m$-последовательностью точность составила, соответственно, 0,96 и 0,95. В быстром режиме медианная точность составила 0,33. Это делает его единственным режимом, точность работы в котором статистически значимо отличается от всех остальных при использовании поправки на множественное сравнение
( $<<0,05)$. Однако в этом режиме у одного испытуемого была достигнута точность 0,96. Такой результат нельзя объяснить случайными причинами, так как это число является результатом ввода 32 команд.

Важной характеристикой ИМК, помимо точности, служит скорость ввода команд. Для режимов с периодом $m$-последовательности в 1 с медианное время, которое требовалось для определения одной команды, составляло 2 с. Для медленного режима этот показатель составил 3,5 c, а для быстрого - 1,2 с.

Интегративным показателем качества работы ИМК, который объединяет точность и скорость выбора команд, служит скорость передачи информации. В данной работе она рассчитана по определению Шеннона, примененного для нейрокомпьютерных интерфейсов [13]. Медианная скорость передачи информации в режимах с базовой и инвертированной последовательностями составила 141 и 142 бит/мин, в медленном режиме 93 бит/мин. Самый низкий показатель скорости передачи информации был достигнут в быстром режиме - 37 бит/мин. Это вызвано низкой точностью выбора команд. Примечательно, что в этом же режиме у единственного пользователя,
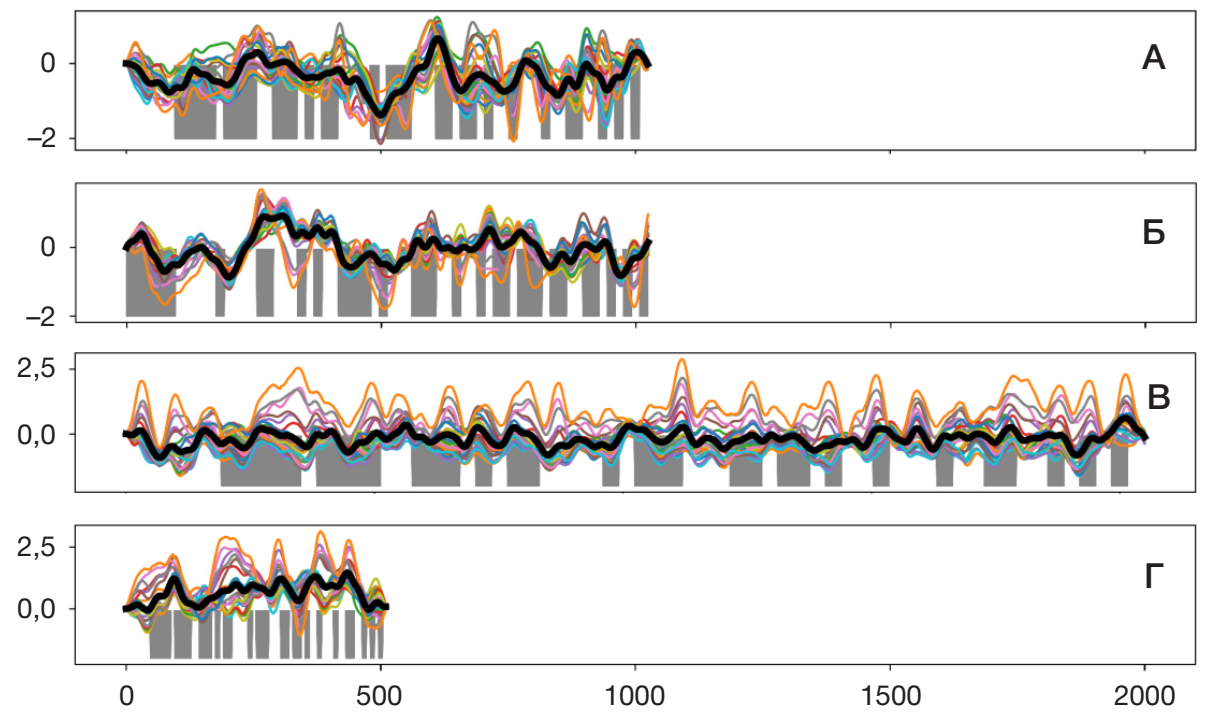

Рис. 2. Полученные в ходе стимуляции усредненные вызванные потенциалы во всех отведениях, приведенные к нулевому сдвигу относительно базовой $m$-последовательности. Серым цветом показана стимульная последовательность. Одна кривая соответствует одному каналу усредненной ЭЭГ. Черным цветом обозначен усредненный между всеми каналами потенциал
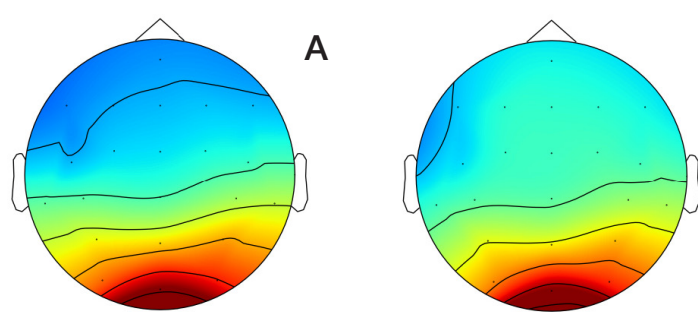

Б
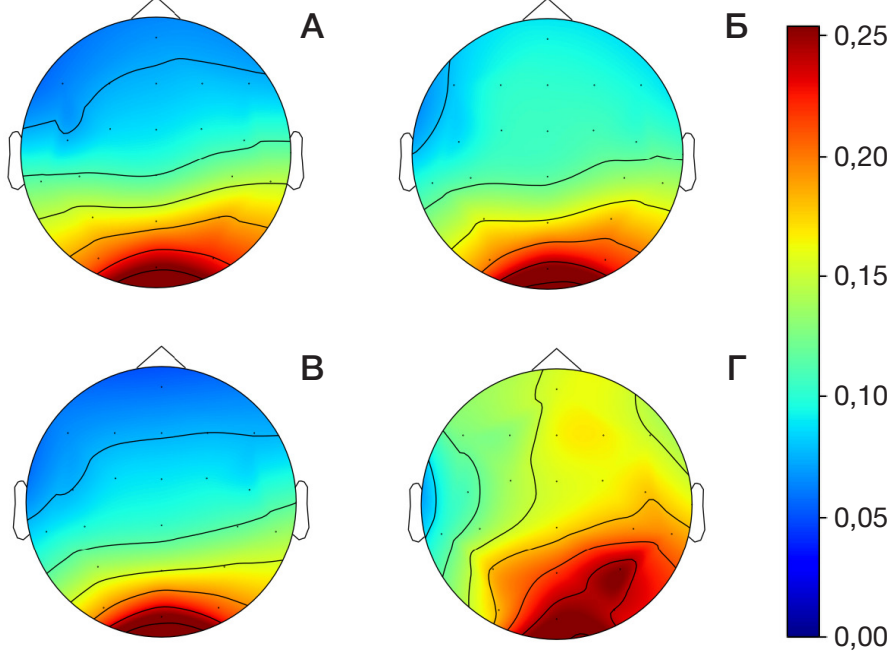

Рис. 3. Усредненные для всех испытуемых и циклов стимуляции карты максимумов корреляционной функции для усредненного в каждом канале вызванного потенциала (рис. 2) и отдельных вызванных потенциалов на одиночные $m$-последовательности. Данный показатель позволяет оценить выраженность потенциала во всех отведениях 
который смог точно вводить команды, достигнут и самый высокий показатель - 287 бит/мин при времени ввода команд, составившем 1 с, и точности, равной 0,96. Скорость передачи информации в режиме с периодом $m$-последовательности в 1 с значимо отличалась от медленного режима $(Z=2,7 ; p=0,019)$.

\section{Форма вызванных потенциалов и топографическое распределение вызванной активности}

На рис. 2 представлена восстановленная для m-последовательности с нулевым сдвигом форма кодированных вызванных потенциалов, усредненных относительно первого бита последовательности.

В качестве количественной характеристики была использована корреляция между усредненным вызванным потенциалом и одиночными потенциалами, соответствующими одиночным $m$-последовательностям На рис. 3 отражены амплитудные карты максимальных значений нормализованных кросс-корреляций между усредненными вызванными потенциалами и реакциями на одиночные последовательности.

Заметно, что вызванные потенциалы демонстрируют максимальную степень сходства между собой в затылочных отведениях. Так, во всех режимах, в которых была достигнута высокая точность выбора команд, самая высокая степень корреляции выражена в отведении $\mathrm{Oz}$ Максимальная корреляция выявлена в 8 каналах: P4 P6, PO3, POz, PO4, O1, O2, Oz. Абсолютные значения максимумов кросс-корреляций не демонстрируют значимых различий между режимами при сравнении в соответствующих каналах. Менее четкая локализация вызванных потенциалов в быстром режиме, возможно, служит одной из причин худших результатов, достигнутых испытуемыми

\section{ОБСУЖДЕНИЕ РЕЗУЛЬТАТОВ}

Результаты исследования ИМК на основе КЗВП позволяют продемонстрировать ряд интересных закономерностей, которые играют роль в построении качественного медицинского нейрокоммуникатора для широкого круга пациентов. В первую очередь, это соотношение между скоростью, точностью ввода и общей скоростью передачи информации в конкретной модификации интерфейса. Очевидно, что главной характеристикой ИМК для пользователя является скорость передачи информации. Полученные данные свидетельствуют о том, что скорость передачи информации у ИМК данного типа в несколько раз выше, чем у традиционного для нейрокоммуникации ИМК-Р300, что создает перспективу внедрения этого типа интерфейсов в клиническую практику. Скорость передачи информации в первых трех режимах находится в привычных рамках для этого типа интерфейсов $[8,14]$. Важным фактом является необычно высокая скорость передачи инсормации в 287 бит/мин, достигнутая одним из пользователей в быстром режиме, который для большинства испытуемых вызвал большие затруднения. Несмотря на уникальность в рамках выборки, этот результат дает повод к разработке ИМК с тонкой подстройкой параметров под индивидуальные особенности пользователей, чтобы найти комбинации параметров (в частности, скорости стимуляции), оптимальные для каждого конкретного пациента. Такой подход может помочь преодолеть известные проблемы переноса лабораторных разработок с участием здоровых испытуемых в клиническую практику [15], а также так называемую ИМК-неграмотность, когда испытуемые оказываются неспособными освоить работу в ИМК [16]. Эти проблемы предлагается решать разными способами, в том числе модификацией тренировочной стадии [17] и индивидуализацией интерфейсов. Тонкая настройка несущей частоты $m$-последовательности и ее периода могла бы помочь найти оптимальные значения, которые максимизируют скорость передачи информации для каждого конкретного пользователя. В частности, подобный подход уже используется для настройки ИМК-P300 [18]. К сожалению, современные мониторы, даже с высокой частотой обновления, не позволяют достаточно гибко настраивать частоту периода стимульной последовательности для ИМК с кодированными вызванными потенциалами. В настоящей работе, к примеру, оказалось невозможным продемонстрировать пользователю последовательность с периодом в 0,8 с. Кажется целесообразным предложить создание специализированного устройства для демонстрации стимулов. Такие попытки для нейроинтерфейсов на основе кодированных вызванных потенциалов уже были предприняты [19], но конкретные представленные реализации обеспечивают низкую скорость передачи информации за счет малого количества стимулов.

\section{ВЫВОДЫ}

Результаты настоящего исследования свидетельствуют о том, что для оптимизации работы испытуемых в ИМК требуется тщательный подбор параметров в зависимости от индивидуальных особенностей пользователей. Было продемонстрировано, что инверсия кодирующей стимульной последовательности не влияет на точность выбора команд испытуемыми, а это свидетельствует о возможности использования режимов прямой и инвертированной стимуляции с равным успехом. В то же время более быстрые режимы работы ИМК с последовательностями в два раза более короткими оказались неоптимальными для большинства испытуемых. Обнаруженные в работе значительные индивидуальные различия показателей точности и скорости передачи информации позволяют предположить, что возможна оптимизация ИМК за счет более тонкой настройки ИМК под конкретного пациента.

\section{Литература}

1. Kaplan AY. Neurophysiological foundations and practical realizations of the brain-machine interfaces in the technology in neurological rehabilitation. Hum Physiol. 2016 Jan 23; 42 (1): 103-10.

2. Wolpaw JR, Birbaumer N, McFarland DJ, Pfurtscheller G, Vaughan TM. Brain-computer interfaces for communication and

control. Clin Neurophysiol [Internet]. 2002 Jun; 113 (6): 767-91. Available from: http://www.ncbi.nlm.nih.gov/pubmed/12048038.

3. Jeunet C, Lotte F, Batail J-M, Philip P, Micoulaud Franchi J-A. Using Recent $\mathrm{BCl}$ Literature to Deepen our Understanding of Clinical Neurofeedback: A Short Review. Neuroscience [Internet]. 
2018 May 15 [cited 2018 Jul 24]; (378): 225-33. Available from: https://www.sciencedirect.com/science/article/pi/S0306452218302045.

4. Akram F, Han H-S, Kim T-S. A P300-based brain computer interface system for words typing. Comput Biol Med [Internet]. 2014 Feb [cited 2014 Jun 1]; (45): 118-25. Available from: http:// www.ncbi.nlm.nih.gov/pubmed/24480171.

5. Yeom SK, Fazli S, Ller KRM, Lee SW. An efficient ERP-based brain-computer interface using random set presentation and face familiarity. PLoS One. 2014; 9 (11): 1-13.

6. Guger C, Allison BZ, Edlinger G. Emerging BCl Opportunities from a Market Perspective. In Springer, Dordrecht; 2014 [cited 2018 Jul 16]: 85-98. Available from: http://link.springer.com/10.1007/97894-017-8996-7_7.

7. McCane LM, Heckman SM, McFarland DJ, Townsend G, Mak JN, Sellers EW, et al. P300-based brain-computer interface (BCl) event-related potentials (ERPs): People with amyotrophic lateral sclerosis (ALS) vs. age-matched controls. Clin Neurophysiol [Internet]. 2015 Nov 1 [cited 2018 Jul 16]; 126 (11): 2124-31. Available from: https://www.sciencedirect.com/science/article/ pii/S138824571500067X.

8. Bin G, Gao X, Wang Y, Li Y, Hong B, Gao S. A high-speed BCl based on code modulation VEP. J Neural Eng. 2011; 8 (2): 025015.

9. Kapeller C, Kamada K, Ogawa H, Prueckl R, Scharinger J, Guger C. An electrocorticographic $\mathrm{BCl}$ using code-based VEP for control in video applications: a single-subject study. Front Syst Neurosci [Internet]. 2014 Aug 7 [cited 2018 Jul 17]; (8): 139. Available from: http://journal.frontiersin.org/article/10.3389/fnsys.2014.00139/ abstract.

10. Spüler M, Rosenstiel W, Bogdan M. Online Adaptation of a c-VEP Brain-Computer Interface $(\mathrm{BCl})$ Based on Error-Related Potentials and Unsupervised Learning. Baumert M, editor. PLoS One [Internet]. 2012 Dec 7 [cited 2018 Jul 17]; 7 (12): e51077. Available from: http://dx.plos.org/10.1371/journal.pone.0051077.

11. Nezamfar H, Salehi SSM, Erdogmus D. Stimuli with opponent colors and higher bit rate enable higher accuracy for C-VEP BCI. In: 2015 IEEE Signal Processing in Medicine and Biology Symposium
(SPMB) [Internet]. IEEE; 2015 [cited 2018 Jul 17]: 1-6. Available from: http://ieeexplore.ieee.org/document/7405476/.

12. Jones E, Oliphant T, Peterson P. \{SciPy\}: Open source scientific tools for \{Python\}. 2001.

13. Yuan $P$, Gao X, Allison B, Wang Y, Bin G, Gao S. A study of the existing problems of estimating the information transfer rate in online brain-computer interfaces. J Neural Eng [Internet]. 2013 Apr 1 [cited $2018 \mathrm{Jul}$ 16]; 10 (2): 026014 . Available from: http:// stacks.iop.org/1741-2552/10/i=2/a=026014?key=crossref.0e89 a1992040af23792558b5b8301c22.

14. Wei Q, Gong H, Lu Z. Grouping modulation with different codes for improving performance in cVEP-based brain-computer interfaces. Electron Lett. 2017 Jan 10; 53 (4): 214-6.

15. Kleih SC, Kaufmann T, Zickler C, Halder S, Leotta F, Cincotti F, et al. Out of the frying pan into the fire-the P300-based BCl faces real-world challenges. Prog Brain Res [Internet]. 2011 Jan 1 [cited 2018 Jul 16]; (194): 27-46. Available from: https://www. sciencedirect.com/science/article/pii/B9780444538154000194.

16. Spezialetti M, Cinque L, Tavares JMRS, Placidi G. Towards EEGbased $\mathrm{BCl}$ driven by emotions for addressing $\mathrm{BCl}-$ Illiteracy: a meta-analytic review. Behav Inf Technol [Internet]. 2018 Aug 3 [cited 2018 Jul 16]; 37 (8): 855-71. Available from: https://www. tandfonline.com/doi/full/10.1080/0144929X.2018.1485745.

17. Jeunet C, Cellard A, Subramanian S, Hachet M, N'Kaoua B, Lotte F. How Well Can We Learn With Standard BCl Training Approaches? A Pilot Study. 2014 [cited 2018 Jul 16]; Available from: https://hal.archives-ouvertes.fr/hal-01052692/.

18. Carabalona R. The Role of the Interplay between Stimulus Type and Timing in Explaining BCl-lliteracy for Visual P300-Based Brain-Computer Interfaces. Front Neurosci [Internet]. 2017 Jun 30 [cited 2018 Jul 16]; (11): 363. Available from: http://journal. frontiersin.org/article/10.3389/fnins.2017.00363/full.

19. Aminaka D, Rutkowski TM. A Sixteen-Command and $40 \mathrm{~Hz}$ Carrier Frequency Code-Modulated Visual Evoked Potential BCl. In Springer, Cham; 2017 [cited 2018 Jul 16]: 97-104. Available from: http://link.springer.com/10.1007/978-3-319-64373-1_10.

\section{References}

1. Kaplan AY. Neurophysiological foundations and practical realizations of the brain-machine interfaces in the technology in neurological rehabilitation. Hum Physiol. 2016 Jan 23; 42 (1): 103-10.

2. Wolpaw JR, Birbaumer N, McFarland DJ, Pfurtscheller G Vaughan TM. Brain-computer interfaces for communication and control. Clin Neurophysiol [Internet]. 2002 Jun; 113 (6): 767-91. Available from: http://www.ncbi.nlm.nih.gov/pubmed/12048038.

3. Jeunet C, Lotte F, Batail J-M, Philip P, Micoulaud Franchi J-A. Using Recent $\mathrm{BCl}$ Literature to Deepen our Understanding of Clinical Neurofeedback: A Short Review. Neuroscience [Internet]. 2018 May 15 [cited 2018 Jul 24]; (378): 225-33. Available from: https://www.sciencedirect.com/science/article/pi/S0306452218302045.

4. Akram F, Han H-S, Kim T-S. A P300-based brain computer interface system for words typing. Comput Biol Med [Internet]. 2014 Feb [cited 2014 Jun 1]; (45): 118-25. Available from: http:// www.ncbi.nlm.nih.gov/pubmed/24480171.

5. Yeom SK, Fazli S, Ller KRM, Lee SW. An efficient ERP-based brain-computer interface using random set presentation and face familiarity. PLoS One. 2014; 9 (11): 1-13.

6. Guger C, Allison BZ, Edlinger G. Emerging BCI Opportunities from a Market Perspective. In Springer, Dordrecht; 2014 [cited 2018 Jul 16]: 85-98. Available from: http://link.springer.com/10.1007/97894-017-8996-7_7.

7. McCane LM, Heckman SM, McFarland DJ, Townsend G, Mak JN, Sellers EW, et al. P300-based brain-computer interface (BCI) event-related potentials (ERPs): People with amyotrophic lateral sclerosis (ALS) vs. age-matched controls. Clin Neurophysiol [Internet]. 2015 Nov 1 [cited 2018 Jul 16]; 126 (11): 2124-31. Available from: https://www.sciencedirect.com/science/article/ pii/S138824571500067X.

8. Bin G, Gao X, Wang Y, Li Y, Hong B, Gao S. A high-speed BCl based on code modulation VEP. J Neural Eng. 2011; 8 (2): 025015. 9. Kapeller C, Kamada K, Ogawa H, Prueckl R, Scharinger J, Guger C. An electrocorticographic $\mathrm{BCl}$ using code-based VEP for control in video applications: a single-subject study. Front Syst Neurosci [Internet]. 2014 Aug 7 [cited 2018 Jul 17]; (8): 139. Available from: http://journal.frontiersin.org/article/10.3389/fnsys.2014.00139/ abstract.

10. Spüler M, Rosenstiel W, Bogdan M. Online Adaptation of a c-VEP Brain-Computer Interface $(\mathrm{BCl})$ Based on Error-Related Potentials and Unsupervised Learning. Baumert M, editor. PLoS One [Internet]. 2012 Dec 7 [cited 2018 Jul 17]; 7 (12): e51077. Available from: http://dx.plos.org/10.1371/journal.pone.0051077.

11. Nezamfar H, Salehi SSM, Erdogmus D. Stimuli with opponent colors and higher bit rate enable higher accuracy for C-VEP BCI. In: 2015 IEEE Signal Processing in Medicine and Biology Symposium (SPMB) [Internet]. IEEE; 2015 [cited 2018 Jul 17]: 1-6. Available from: http://ieeexplore.ieee.org/document/7405476/.

12. Jones E, Oliphant T, Peterson P. \{SciPy\}: Open source scientific tools for \{Python\}. 2001.

13. Yuan P, Gao X, Allison B, Wang Y, Bin G, Gao S. A study of the existing problems of estimating the information transfer rate in online brain-computer interfaces. J Neural Eng [Internet]. 2013 Apr 1 [cited 2018 Jul 16]; 10 (2): 026014. Available from: http:// stacks.iop.org/1741-2552/10/i=2/a=026014?key=crossref.0e89 a1992040af23792558b5b8301c22.

14. Wei Q, Gong H, Lu Z. Grouping modulation with different codes for improving performance in cVEP-based brain-computer interfaces. Electron Lett. 2017 Jan 10; 53 (4): 214-6.

15. Kleih SC, Kaufmann T, Zickler C, Halder S, Leotta F, Cincotti F, et al. Out of the frying pan into the fire-the P300-based BCl faces real-world challenges. Prog Brain Res [Internet]. 2011 Jan 1 
[cited 2018 Jul 16]; (194): 27-46. Available from: https://www sciencedirect.com/science/article/pii/B9780444538154000194.

16. Spezialetti M, Cinque L, Tavares JMRS, Placidi G. Towards EEGbased $\mathrm{BCl}$ driven by emotions for addressing $\mathrm{BCl}$-Illiteracy: a meta-analytic review. Behav Inf Technol [Internet]. 2018 Aug 3 [cited 2018 Jul 16]; 37 (8): 855-71. Available from: https://www. tandfonline.com/doi/full/10.1080/0144929X.2018.1485745.

17. Jeunet C, Cellard A, Subramanian S, Hachet M, N'Kaoua B, Lotte F. How Well Can We Learn With Standard BCl Training Approaches? A Pilot Study. 2014 [cited 2018 Jul 16]; Available from: https://hal.archives-ouvertes.fr/hal-01052692/.
18. Carabalona R. The Role of the Interplay between Stimulus Type and Timing in Explaining BCl-Illiteracy for Visual P300-Based Brain-Computer Interfaces. Front Neurosci [Internet]. 2017 Jun 30 [cited 2018 Jul 16]; (11): 363. Available from: http://journal. frontiersin.org/article/10.3389/fnins.2017.00363/full.

19. Aminaka D, Rutkowski TM. A Sixteen-Command and $40 \mathrm{~Hz}$ Carrier Frequency Code-Modulated Visual Evoked Potential BCl. In Springer, Cham; 2017 [cited 2018 Jul 16]: 97-104. Available from: http://link.springer.com/10.1007/978-3-319-64373-1_10. 